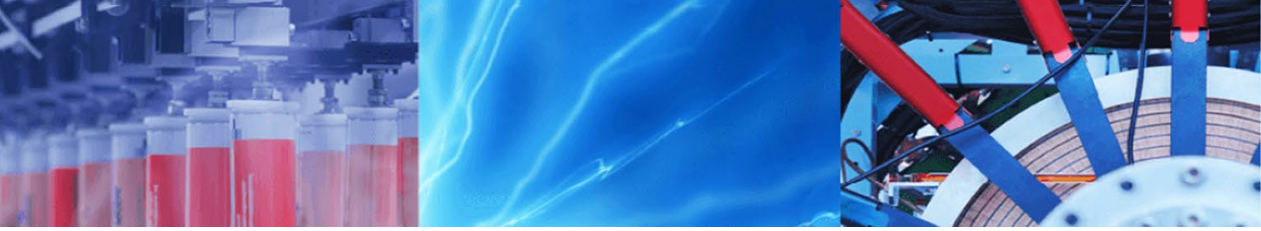

Research Article

\title{
Fixed time terminal sliding mode trajectory tracking design for a class of nonlinear dynamical model of air cushion vehicle
}

\author{
Hamede Karami $^{1} \cdot$ Reza Ghasemi $^{1}$ (D)
}

Received: 28 October 2019 / Accepted: 7 December 2019 / Published online: 17 December 2019

(c) Springer Nature Switzerland AG 2019

\begin{abstract}
This manuscript proposes a robust fixed time terminal sliding mode prototype for trajectory tracking of the nonlinear dynamics of an under-actuated air cushion vehicle. Nonlinearity, external disturbances, internal uncertainties and unmodeled dynamics are the main difficulties that an amphibious vehicle is faced with in its maneuver. The main contribution of the proposed methodology is to overcome these problems based on both the guaranteed stability in sense of Lyapunov and the fixed time tracking error even if the initial values are changed. Robustness against uncertainties and disturbances, fixed time convergence of tracking error to zero are other merits of the proposed approach. The simulation results demonstrate the effectiveness and superiority of suggested scheme.
\end{abstract}

Keywords Sliding mode control · Fixed time $\cdot$ Nonlinear control $\cdot$ Trajectory tracking $\cdot$ Hovercraft $\cdot$ Air cushion vehicle

\section{Introduction}

\subsection{Background and motivations}

Air cushion vehicle (ACV) is an under-actuated electromechanical system. The amphibious hovercraft is located on air cushion therefore, it can move on every surface and consequently its used in many applications [1], but the control of hovercraft is so complex, because of low friction, high speed, second order nonholonomic constraint which makes its motion restrict, actuators generate forces only in heading path, dynamic coupling exist among states, external disturbances can affect its motion and directly compensate for side-external disturbance is impossible [1, 2]. Therefore, controller must be designed so that overcome aforementioned challenges. Sliding mode control (SMC) has been widely used to control various systems in recent decades [3-7], this methodology of control has some notable merits for control engineers such as robustness against disturbances, uncertainties and parameter variations, high accuracy, fast dynamic response, simplicity of computations, significant transient performance and guaranteed stability $[8,9]$. The main disadvantages of SMC are chattering effect due to discontinues control action and infinity settling time. Chattering phenomena can hurt the system actuators and sensors. These problems can be solved by high order SMC [10-13]. Trajectory tracking control is one of the most attractive and challenging tasks of control for a long time [14-16].

The question may be inspired control engineers, how can do tracking control and stabilizing hovercraft in a fixed time, whether initial values are changed despite all of aforementioned SMC merits.

\subsection{Brief survey}

$A C V$ is a high performance and high usage vehicle, but control of ACV is difficult, the main problems of ACV control are point stabilization, path following and trajectory tracking [17]. Two control methods have been applied on hovercraft so far, classic and intelligent.

Reza Ghasemi, r.ghasemi@qom.ac.ir | 'Department of Electrical Engineering, University of Qom, Qom, Iran. 
In [18-21] fuzzy controllers derived on hovercraft, although the methods are simple, fuzzy method has problems with systematic analysis and design. In [22] the controller has been designed based on a neural network and applied to a hovercraft dynamic in which the actuator has not been modeled, and the use of the neural network has not led to a globally stability, it can't follow the desired path completely. In [23] a radial basis function neural networks controller has been implemented in a number of hovercraft coordinates. The effect of external disturbances and friction have not taken into account and the dynamic model is a simple model, and also the time delay of communication between devices has been ignored. Therefore, it can generally be noted that intelligent controllers, are very complex decision making processes and fuzzy rules are based on the knowledge of experts, which is not always available. In addition, great set of rules need great time to compute and adjust.

The classical control methods that carried out on hovercraft so far include a Proportional, Integrated, Derivative(PID), adaptive, backstepping, open loop control, linear and nonlinear Lyapunov-based, linear regulator controllers, in which the effect of external disturbances and frictions are ignored, and also they are not robust against them [24-28]. Other controllers such as feedback linearization, state feedback, etc. don't have high accuracy for this kind of extreemly nonlinear dynamics and may not be applicable because of the existance of discountinus functions in the dynamics [29-33].

Human-centered tracking system for a hovercraft based on terminal sliding mode control is applied in [34] the chattering-free and full order TSM has solved chattering and singularity problems then its combined with radial basis function neural networks to deal with the nonlinearity and uncertainty of hovercraft's model and it can convergs velocities and position tracking errors at finite-time.

The path following and trajectory tracking tasks in a hovercraft physical model are controlled by the backstepping controller in [35], the friction coefficients and disturbance dynamic estimators are introduced, global practical stability is achieved and actuations are remained bounded with respect to the position error.

In [36-42], some combinations of sliding mode with other controllers on hovercraft and other systems are presented. Although they are robust against disturbances, they haven't been able to stabilize system at a fixed time.

\subsection{Contribution}

Motivated by mentioned considerations, this manuscript proposed a controller which is robust against disturbances and uncertainties, reduces chattering, converges tracking error to zero and guarantees the overall close loop stability. The control law is designed based on lyapunove stability. The main innovations of this paper in comparison with the related references which applied on hovercraft are as follows:

- A robust fixed time terminal nonlinear SMC scheme is designed for tracking control of the ACV, which isn't designed in this methodology for the ACV before.

- To get the fixed time stability even if the initial values are changed, the sliding surface is designed in new form, which isn't used in sliding mode control algorithms of the hovercraft.

- The chattering reduction is done by using the sigmoid function.

- The dynamical model structure which is used in this paper induces lateral forces on the ACV depending on the torque. In other models, thrust and torque are independent. Therefore, the nonlinearity properties of hovercraft dynamic are fully modeled based on its interaction.

\subsection{Paper organization}

The remainder of this paper is structured as follows, Sect. 2 describes some preliminaries such as notations which are used in this paper, problem formulation which demonstrates vehicle modeling contains a description of nonlinear model for hovercraft and some lemmas which are required for controller design and express the rest of the paper. Section 3 presents fixed time terminal sliding mode control design and its structure. Simulation results are presented in Sect. 4. Finally, Sect. 5 provides a brief conclusion.

\section{Preliminaries}

\subsection{Notations}

This section is represented for easy access and better description of equations and also avoidance of repetition. Table 1 shows this paper symbols and their descriptions.

\subsection{Problem formulation}

To better comprehension of the problem first of all, the following lemma [43] should be expressed.

Lemma 1 Consider a system such as

$\dot{x}=-\alpha x^{\frac{m}{n}}-\beta x^{\frac{p}{q}}, x(0)=x_{0}$ 
Table 1 Symbol table

\begin{tabular}{|c|c|c|c|}
\hline Symbol & Description & Symbol & Description \\
\hline$u$ & Longitudinal velocity & $b_{T}$ & Force scaling coefficient \\
\hline$v$ & Lateral velocity & $k_{1}, k_{2}$ & Switching gain \\
\hline$\theta$ & Rudder angle & $e_{u}, e_{v}$ & Velocity errors \\
\hline$r$ & Angular velocity & $u_{d}, v_{d}, x_{d}, y_{d}$ & Desired parameters \\
\hline$T$ & Trust force & $\left\{d_{u_{0}}, d_{v_{0}}, d_{r_{0}}, d_{u}, d_{v}, d_{r}\right\}$ & Friction coefficients \\
\hline$m$ & Mass of hovercraft & $I_{x}, I_{y}$ & Saturation constants \\
\hline$J$ & Inertia moment & $k_{x}, k_{y}$ & Controller gains \\
\hline$a$ & $\begin{array}{l}\text { The arm length from the center of } \\
\text { mass to the rudder surface }\end{array}$ & {$\left[\begin{array}{cc}\cos \theta & \sin \theta \\
-\sin \theta & \cos \theta\end{array}\right]$} & Rotational matrix \\
\hline$x_{e}, y_{e}$ & Position tracking error & & \\
\hline
\end{tabular}

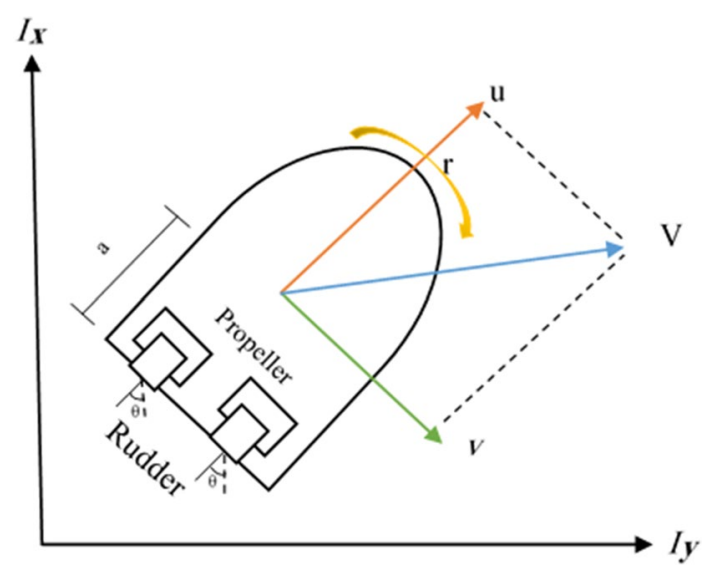

Fig. 1 Sketch of ACV model

where $\alpha, \beta$ are positive, $m, n, p, q>0$ and odd which $m>n, p<q$, therefore, the equilibrium point of (1) is fixed time stable and fixed time upper bound is as follows

$T_{\max }=\frac{1}{\alpha} \frac{n}{m-n}+\frac{1}{\beta} \frac{q}{q-p}$

\section{And it is independent of initial states.}

The ACV model (Fig. 1) is considered as a rigid body in a 2-D space and the following equations are used as kinematics and dynamics [45].

$$
\left\{\begin{array}{l}
\dot{x}=\cos \theta u-\sin \theta v \\
\dot{y}=\sin \theta u+\cos \theta v \\
\dot{u}=-m^{-1} d_{u_{0}} \operatorname{sign} u-m^{-1} d_{u} u+m^{-1} b_{T} T \cos \theta+v r \\
\dot{v}=-m^{-1} d_{v_{0}} \operatorname{sign} v-m^{-1} d_{v} v+m^{-1} b_{T} T \sin \theta-u r \\
\dot{r}=-J^{-1} d_{r_{0}} \operatorname{sign} r-J^{-1} d_{r} r-J^{-1} a b_{T} T \sin \theta
\end{array}\right.
$$

The parameters of (3) are described in Table 1. Velocity errors can be defined as $\left\{\begin{array}{l}e_{u}=u-u_{d} \\ e_{v}=v-v_{d}\end{array}\right.$

where $u_{d}$ and $v_{d}$ are desired longitudinal and lateral velocity, and the time derivative of (4) by substituting (3) is obtained as

$\left\{\begin{array}{l}\dot{e}_{u}=-m^{-1} d_{u_{0}} \operatorname{sign} u-m^{-1} d_{u} u+m^{-1} b_{T} T \cos \theta+v r-\dot{u}_{d} \\ \dot{e}_{v}=-m^{-1} d_{v_{0}} \operatorname{sign} v-m^{-1} d_{v} v+m^{-1} b_{T} T \sin \theta-u r-\dot{v}_{d}\end{array}\right.$

The $u_{d}$ and $v_{d}$ are considered as

$\left[\begin{array}{l}u_{d} \\ v_{d}\end{array}\right]=\left[\begin{array}{cc}\cos \theta & \sin \theta \\ -\sin \theta & \cos \theta\end{array}\right]\left[\begin{array}{l}\dot{x}_{d}+I_{x} \tanh \left(\frac{-k_{x}}{I_{x}} x_{e}\right) \\ \dot{y}_{d}+I_{y} \tanh \left(\frac{-k_{y}}{l_{y}} y_{e}\right)\end{array}\right]$

where $k_{x}, k_{y}, I_{x}, I_{y}>0$ and $x_{e}=x-x_{d}, y_{d}=y-y_{d}$

Theorem 1 If the errors of velocity $e_{u}$ and $e_{v}$ in (6) converge to zero, then it is ensured that the errors of position tracking $\left(x_{e} y_{e}\right)$ asymptotically converge to origin [15].

Proof From kinematics equations in (3), the following equation is obtained.

$\left[\begin{array}{l}u \\ v\end{array}\right]=\left[\begin{array}{cc}\cos \theta & \sin \theta \\ -\sin \theta & \cos \theta\end{array}\right]\left[\begin{array}{l}\dot{x} \\ \dot{y}\end{array}\right]$

With substituting (6) and (7) to (4) velocity errors are obtained as

$\left[\begin{array}{l}e_{u} \\ e_{v}\end{array}\right]=\left[\begin{array}{cc}\cos \theta & \sin \theta \\ -\sin \theta & \cos \theta\end{array}\right]\left[\begin{array}{l}\dot{x}_{e}-I_{x} \tanh \left(\frac{-k_{x}}{I_{x}} x_{e}\right) \\ \dot{y}_{e}-I_{y} \tanh \left(\frac{-k_{y}}{I_{y}} y_{e}\right)\end{array}\right]$

If velocity errors $\left(e_{u}, e_{v}\right)$ converge to zero, thus Eq. (9) is obtained, because rotational matrix isn't singular. 


$$
\left\{\begin{array}{l}
\dot{x}_{e}=I_{x} \tanh \left(\frac{-k_{x}}{I_{x}} x_{e}\right) \\
\dot{y}_{e}=I_{y} \tanh \left(\frac{-k_{y}}{I_{y}} y_{e}\right)
\end{array}\right.
$$

To prove the convergence position errors to zero, the Lyapunov function is considered as

$V_{1}=\frac{1}{2} x_{e}^{2}+\frac{1}{2} y_{e}^{2}$

The time derivative of $V_{1}$ is

$\dot{V}_{1}=x_{e} \dot{x}_{e}+y_{e} \dot{y}_{e}=-l_{x} x_{e} \tanh \left(\frac{k_{x}}{I_{x}} x_{e}\right)-I_{y} y_{e} \tanh \left(\frac{k_{y}}{l_{y}} y_{e}\right)$

As tanh is an odd function and controller and saturation coefficients are positive therefore, $\dot{V}_{1}$ is negative and position errors converge to zero.

The model and the control conditions make the following assumptions.

Assumption 1 For simplicity, the explicit time dependence of position and uncertainties is omitted.

Assumption 2 The states of the system are fully measureable.

\section{Controller design methodology}

This section presents the proposed design of fixed time terminal sliding mode controller, the main target of this part is to design robust control laws for the surge and sway velocity therefore, the position of the ACV can track a desired target. Figure 2 depicts a diagram of the ACV tracking control design phases.

\subsection{Controller structure}

The target of the paper is trajectory tracking and fixed time stability even though initial conditions are changed. Therefore, the sliding surfaces are considered as follows.

$\left\{\begin{array}{l}S_{1}=e_{u}+\alpha \int_{0}^{t} \operatorname{sign}\left(e_{u}\right)\left|e_{u}\right|^{\frac{m}{n}} d \tau+\beta \int_{0}^{t} \operatorname{sign}\left(e_{u}\right)\left|e_{u}\right|^{\frac{p}{a}} d \tau \\ S_{2}=e_{v}+\alpha \int_{0}^{t} \operatorname{sign}\left(e_{v}\right)\left|e_{v}\right|^{\frac{m}{n}} d \tau+\beta \int_{0}^{t} \operatorname{sign}\left(e_{v}\right)\left|e_{v}\right|^{\frac{p}{a}} d \tau\end{array}\right.$

where $m>n, 0.5<\frac{p}{q}<1$ and $m, n, p, q$ are integers which are positive, $\alpha, \beta, \lambda$ are positive.

To obtain finite time terminal sliding mode, the time derivative of sliding surfaces should be constrained by the following conditions.

$\left\{\begin{array}{l}\dot{S}_{1}=-\alpha \operatorname{sign}\left(S_{1}\right)\left|S_{1}\right|^{\frac{m}{n}}-\beta \operatorname{sign}\left(S_{1}\right)\left|S_{1}\right|^{\frac{p}{q}} \\ \dot{S}_{2}=-\alpha \operatorname{sign}\left(S_{2}\right)\left|S_{2}\right|^{\frac{m}{n}}-\beta \operatorname{sign}\left(S_{2}\right)\left|S_{2}\right|^{\frac{p}{q}}\end{array}\right.$

By equalizing the time derivative of (12) to (13), one obtains

$\left\{\begin{array}{l}\dot{e}_{u}=-\alpha \operatorname{sign}\left(e_{u}\right)\left|e_{u}\right|^{\frac{m}{n}}-\beta \operatorname{sign}\left(e_{u}\right)\left|e_{u}\right|^{\frac{p}{q}} \\ \dot{e}_{v}=-\alpha \operatorname{sign}\left(e_{v}\right)\left|e_{v}\right|^{\frac{m}{n}}-\beta \operatorname{sign}\left(e_{v}\right)\left|e_{v}\right|^{\frac{p}{q}}\end{array}\right.$

From Lemma 1 and the Eqs. (13) and (14), the convergence conditions of sliding surfaces and errors to the origin in a fixed time are obtained.

Control inputs are proposed as [44] and (9) that consist of two sections, $U_{e q}$ shows the equivalent term to eliminate the certain term and $U_{r}$ demonstrates the reaching one to decrease the uncertainties [45].

$\theta=\theta_{e q}+\theta_{r}$

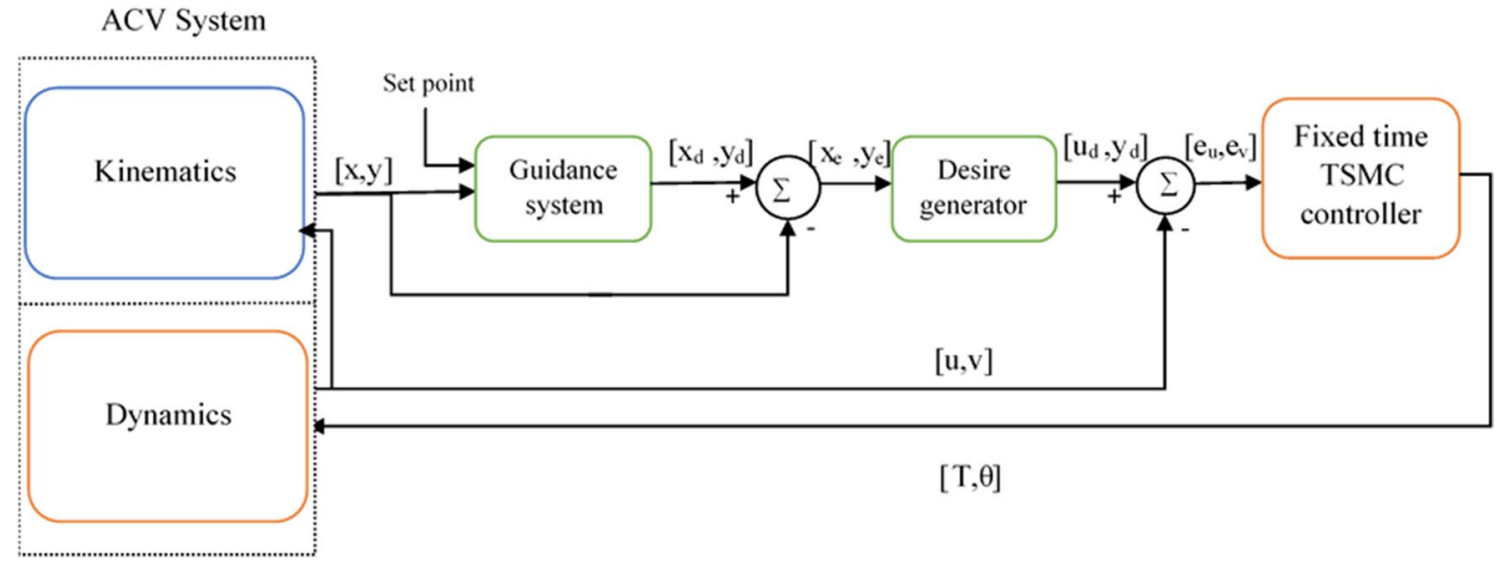

Fig. 2 A block diagram of the ACV's tracking controller design 
$T=T_{\text {eq }}+T_{r}$

Equivalent thrust control input can be selected as follows

$T_{e q}=\frac{1}{m^{-1} b_{T}}\left(\begin{array}{l}m^{-1} d_{u 0} \operatorname{signu}+m^{-1} d_{u} u-v r+\dot{u}_{d}-\alpha \operatorname{sign}\left(e_{u}\right)\left|e_{u}\right|^{\frac{m}{n}} \\ -\beta \operatorname{sign}\left(e_{u}\right)\left|e_{u}\right|^{\frac{p}{q}}-\alpha \operatorname{sign}\left(S_{1}\right)\left|S_{1}\right|^{\frac{m}{n}}-\beta \operatorname{sign}\left(S_{1}\right)\left|S_{1}\right|^{\frac{p}{q}}\end{array}\right)$

$$
\left\{\begin{aligned}
\dot{S}_{1} & =-m^{-1} d_{u_{0}} \operatorname{sign} u-m^{-1} d_{u} u+m^{-1} b_{T} T \cos \theta+v r-\dot{u}_{d} \\
& +\alpha \operatorname{sign}\left(e_{u}\right)\left|e_{u}\right|^{\frac{m}{n}}+\beta \operatorname{sign}\left(e_{u}\right)\left|e_{u}\right|^{\frac{p}{q}} \\
\dot{S}_{2} & =\lambda\left(-m^{-1} d_{v_{0}} \operatorname{sign} v-m^{-1} d_{v} v+m^{-1} b_{T} T \sin \theta-u r-\dot{v}_{d}\right) \\
& +\alpha \operatorname{sign}\left(e_{v}\right)\left|e_{v}\right|^{\frac{m}{n}}+\beta \operatorname{sign}\left(e_{v}\right)\left|e_{v}\right|^{\frac{p}{q}}
\end{aligned}\right.
$$

And rudder angle is obtained as

$\theta_{e q}=\frac{1}{m^{-1} b_{T} T}\left(\begin{array}{l}m^{-1} d_{v 0} \operatorname{signv}+m^{-1} d_{v} v+u r+\dot{v}_{d}-\alpha \operatorname{sign}\left(e_{v}\right)\left|e_{v}\right|^{\frac{m}{n}} \\ -\beta \operatorname{sign}\left(e_{v}\right)\left|e_{v}\right|^{\frac{p}{q}}-\alpha \operatorname{sign}\left(S_{2}\right)\left|S_{2}\right|^{\frac{m}{n}}-\beta \operatorname{sign}\left(S_{2}\right)\left|S_{2}\right|^{\frac{p}{q}}\end{array}\right)$

Reaching inputs in order to traditional symbolic function considered as sigmoid which can reduce chattering effect [42]

$\theta_{r}=-k_{1} \operatorname{sig}\left(S_{1}\right)$

$T_{r}=-k_{2} \operatorname{sig}\left(S_{2}\right)$

The defenition of sigmoid function is

$\operatorname{sig}(S)=\frac{1}{1+\exp (-\psi(S-\sigma))}$

where $\psi, \sigma$ are positive constant.

\subsection{Stability and convergence time analysis}

Theorem 2 Consider hovercraft dynamics given in (3) satisfying Assumptions 1 and 2. Then the sliding surface mentioned in Eq. (12) and control inputs proposed as (17) to (20) makes the closed loop system asymptotically stable, tracking errors and sliding surface converge to zero furthermore all signals in the closed loop system will be bounded.

Proof To prove the stability of closed loop system, the Lyapunov function candidate is as follows.

$V_{2}=\frac{1}{2} S_{1}^{2}+\frac{1}{2} S_{2}^{2}$

The time derivative of Lyapunov function should be satisfied following inequality.

$\dot{V}_{2}=S_{1} \dot{S}_{1}+S_{2} \dot{S}_{2} \leq-\eta_{1}\left|S_{1}\right|-\eta_{2}\left|S_{2}\right|$

with time derivative of (12) and substituting (3) in it
By substituting control inputs (17-20) in (24) and considering (13) then substituting in time derivative of Lyapunov function therefore, Eq. (25) is obtained.

$$
\begin{aligned}
\dot{V}_{2}= & S_{1}\left(-\alpha \operatorname{sign}\left(S_{1}\right)\left|S_{1}\right|^{\frac{m}{n}}-\beta \operatorname{sign}\left(S_{1}\right)\left|S_{1}\right|^{\frac{p}{q}}\right) \\
& +S_{2}\left(-\alpha \operatorname{sign}\left(S_{2}\right)\left|S_{2}\right|^{\frac{m}{n}}-\beta \operatorname{sign}\left(S_{2}\right)\left|S_{2}\right|^{\frac{p}{q}}\right)
\end{aligned}
$$

Then (25) can be rewritten as

$\dot{V}_{2}=-\alpha\left|S_{1}\right|^{\frac{m}{n}+1}-\beta\left|S_{1}\right|^{\frac{p}{q}+1}-\alpha\left|S_{2}\right|^{\frac{m}{n}+1}-\beta\left|S_{2}\right|^{\frac{p}{q}+1}$

By some math manupulation Eq. 26 can be rewritten as

$\dot{V}_{2} \leq-A V_{2}^{\frac{m+n}{2 n}}-B V_{2}^{\frac{p+q}{2 q}} \leq-A V_{2}^{\delta_{1}}-B V_{2}^{\delta_{2}}$

It's clear that (26) is negative and an $\eta$ is exists which can satisfy inequality (23), therefore stability of fixed time sliding mode control is proven. This completes the proof.

Theorem 3 Consider the ACV model (3), with the control inputs selected as (15) and (16), then the states converge to origin in fixed time and for each sliding surfaces upper bound of settling time $\left(T_{s}\right)$ obtain as equation $[43,44]$.

Proof The time which is set for Eq. (24) is T (S (0)), from Lemma 1. The time which system (3) can reach the sliding surface satisfies:

$\lim _{S_{1,2}(0) \rightarrow \infty} T\left(S_{1,2}(0)\right) \leq \frac{1}{A\left(\delta_{1}-1\right)}+\frac{1}{B\left(1-\delta_{2}\right)} \leq T_{\max }^{\prime}$

Therefore, the system reaches the sliding surfaces in a fixed time which the upper bound of it is $T_{\text {max }}^{\prime}$.

To present the upper bound of state errors, Lyapunov stability of errors should be proven, thus Lyapunov function candidate considered as follows 
$V_{3}=\frac{1}{2}\left(e_{u}^{2}+e_{v}^{2}\right)$

The time derivative of (29) is

$$
\begin{aligned}
\dot{V}_{3}= & e_{u} \dot{e}_{u}+e_{v} \dot{e}_{v}=-\alpha\left(\left|e_{u}\right|^{\frac{m}{n}+1}+\left|e_{v}\right|^{\frac{m}{n}+1}\right)-\beta\left(\left|e_{u}\right|^{\frac{p}{q}+1}+\left|e_{v}\right|^{\frac{p}{q}+1}\right) \\
& \leq-A V_{3}^{\delta_{1}}-B V_{3}^{\delta_{2}}
\end{aligned}
$$

According to Lemma1 and (30), Time of error function converging to origin can be expressed as

$\lim _{e_{u, v}(0) \rightarrow \infty} T\left(e_{u, v}(0)\right) \leq \frac{1}{A\left(\delta_{1}-1\right)}+\frac{1}{B\left(1-\delta_{2}\right)} \leq T_{\max }^{\prime}$

From (28) to (31) the upper bound of system's settling time satisfies

$$
\begin{aligned}
\lim _{\substack{e_{u, v}(0) \rightarrow \infty \\
S_{1,2}(0) \rightarrow \infty}} T_{s} \leq & \lim _{\substack{e_{u, v}(0) \rightarrow \infty \\
S_{1,2}(0) \rightarrow \infty}}\left(T\left(e_{u, v}(0)\right)+T\left(S_{1,2}(0)\right)\right) \\
& \leq \frac{2}{A\left(\delta_{1}-1\right)}+\frac{2}{B\left(1-\delta_{2}\right)} \leq 2 T_{\text {max }}^{\prime}
\end{aligned}
$$

The Eq. (32) presents that states of system (3) converge in a fixed time which it's upper bound is $2 T_{\max }^{\prime}$. This time can be set based on problem requirements.

\section{Simulation results}

As an illustration of this paper suggested method, the ACV is simulated using friction coefficients and parameters of $[44,46]$. The mass of ACV is $0.585(\mathrm{~kg})$, length of the arm is $0.14(\mathrm{~m})$ and inertia is $0.01\left(\mathrm{~kg} \mathrm{~m}^{2}\right)$. Table 2 presents the controller parameters.

As it is explained before $m>n, 0.5<\frac{p}{q}<1$ and $m, n, p, q$ are integers which are positive, $\alpha, \beta, \lambda k_{x}, k_{y}, I_{x}, I_{y}, \psi, \sigma$ are positive constant therefore, the best values for better controller behavior are obtained using the trial and error method.

Table 2 The controller parameters

\begin{tabular}{lcll}
\hline Parameter & Value & Parameter & Value \\
\hline$p$ & 2 & $\beta$ & 2 \\
$q$ & 3 & $k_{1}$ & 10 \\
$m$ & 3 & $k_{2}$ & 0.1 \\
$n$ & 2 & $\lambda$ & 0.9 \\
$a$ & 4 & $\psi$ & 1 \\
$k_{x}$ & 16 & $\sigma$ & 1 \\
$k_{y}$ & 64 & $I_{x}$ & 8 \\
$l_{y}$ & 32 & & \\
\hline
\end{tabular}

SN Applied Sciences
Control laws which are mentioned before, applied to the model and following figures are the results. The time value of simulation is around $100 \mathrm{~s}$ for the ordinary PCs. It should be noticed that a linear system with sinusoidal input is given to the controller as the desired tracking system.

The sliding surfaces of controller are depicted in Fig. 3.

Both Sliding surfaces in a short times converge to origin. Now in following figures ACV states and the desired values are displayed. According to Eq. (6), desired values defined for $x, y$, $u$ and $v$ only, therefore tracking simulation is shown for them (Fig. 4).

By the reference system which is considered for tracking, the steady state velocity components values are in range $[-0.1,0.1]$ (Fig. 5).
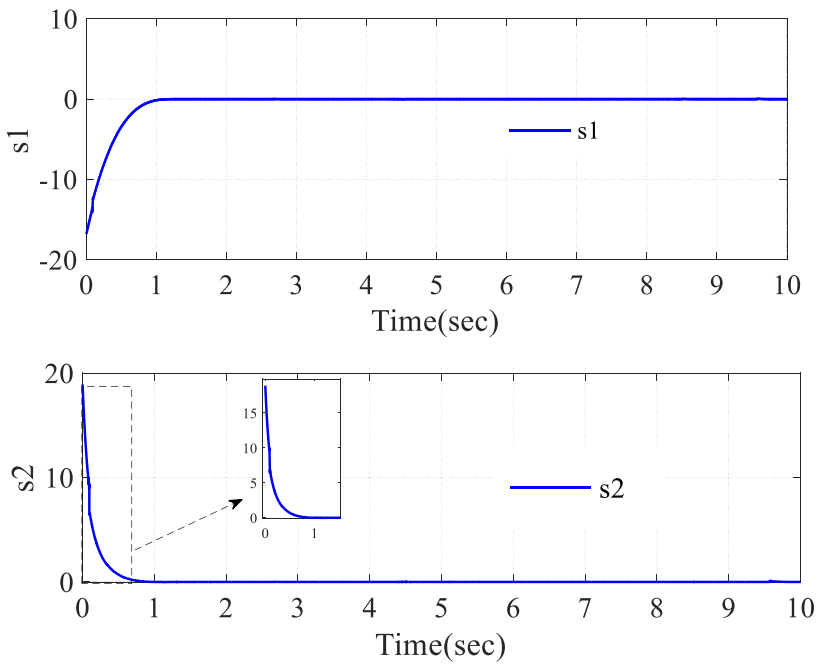

Fig. 3 Sliding surfaces
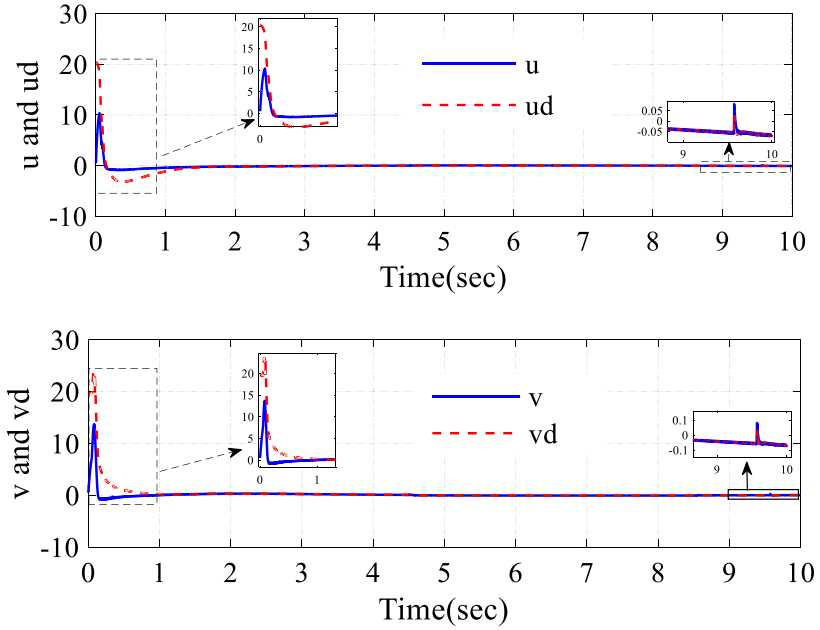

Fig. 4 Velocities tracking of desired references 

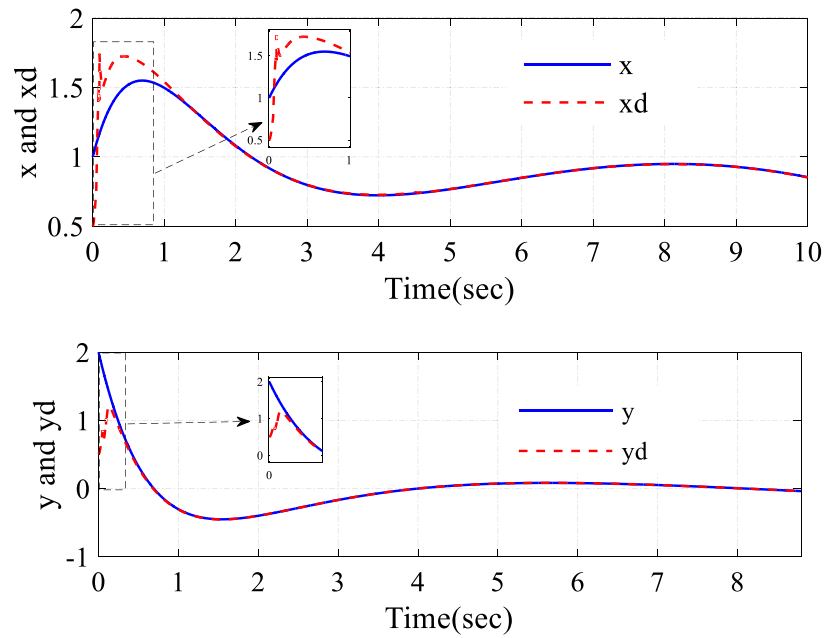

Fig. 5 Position tracking of desired references
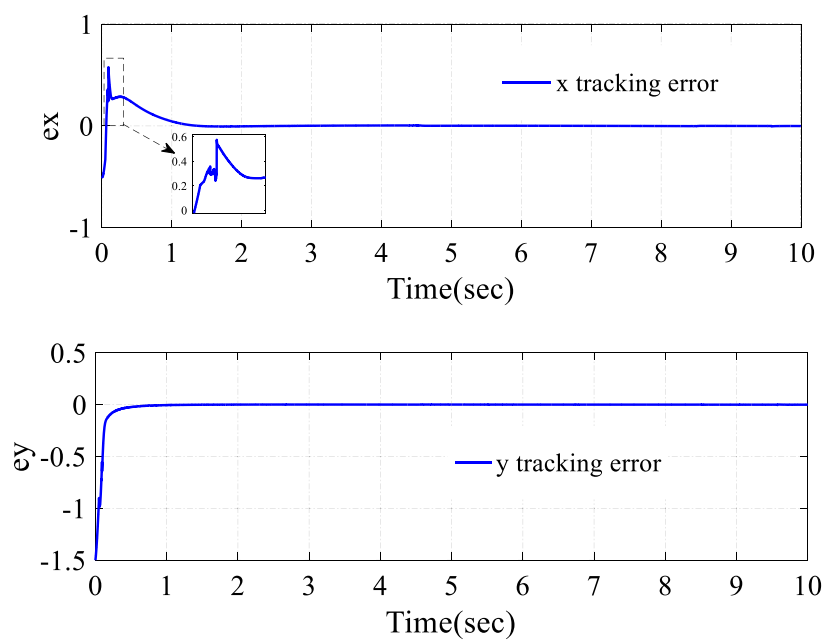

Fig. 6 Position tracking error

As it's clear from simulation results, the performance of the proposed method of tracking is amazing, after split seconds system can track desired reference (Fig. 6).

The tracking errors of position and velocity before $1 \mathrm{~s}$ converge to zero (Fig. 7).

As it is expressed before, a linear system with sinusoidal input considered as the desired tracking system of controller, then at the split time the controller could make system asymptotically stable and sliding surfaces and states tracking errors converge to the origin.

\section{Conclusion}

In this paper, fixed time terminal sliding mode controller is designed ACV to track the predefined trajectories. This technique is robust against disturbances and uncertainties
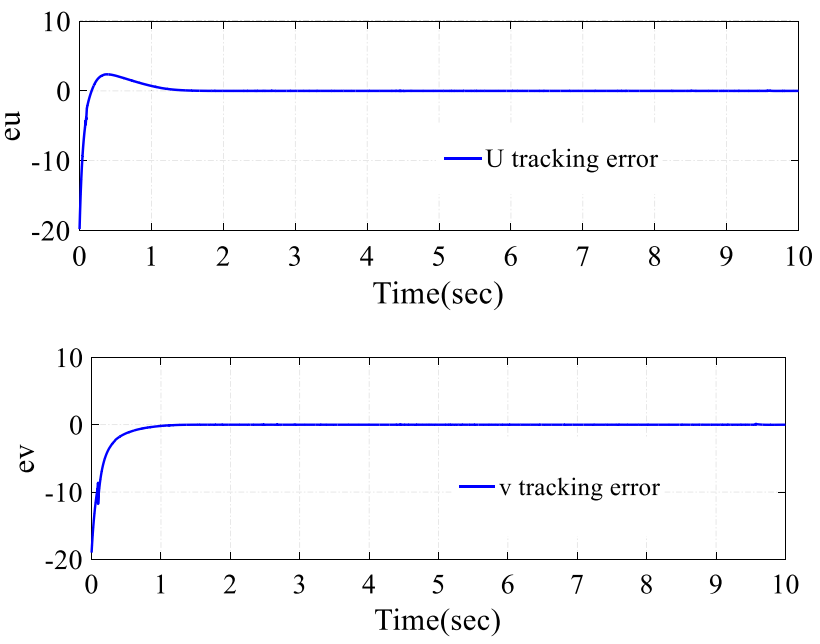

Fig. 7 Velocity tracking error

but the main target of the paper is to persuade the ACV to track the desired reference and stabilizing at the fixed time even if the initial conditions are changed. The settling time can be estimated in advance and the chattering is reduced easily. Asymptotic stability of overall close loop system is proven and by making velocity error to zero, ACV tracks desired path and position tracking error converges to zero too. Simulation results demonstrate efficiency and superiority of proposed method. In this research states are assumed accessible, in future works it is better to design a neural observer for estimating inaccessible states, disturbance and uncertainties. The boundedness of stability time may cause the enhancement of control effort therefore, it is better to have a trade-off between settling time and energy. The extension of this method to higher order systems can be considered for future studies.

\section{Compliance with the ethical standards}

Conflict of interest The authors (Hemede Karami, Reza Ghasemi) declare that they have no conflict of interest.

\section{References}

1. Jeong S, Chwa D (2017) Coupled multiple sliding-mode control for robust trajectory tracking of hovercraft with external disturbances. IEEE Trans Ind Electron 65(5):4103-4113

2. Jeong S, Eom M, Chwa D (2015) Disturbance-estimation-based hierarchical sliding mode control of hovercraft with wind disturbance. In: 2015 15th international conference on control, automation and systems (ICCAS). IEEE, pp 532-537

3. Torres JZ, Cieslak J, Henry D, Davila J (2019) A sliding mode control in a backstepping setup for rendezvous mission on a circular orbit. In: 8th European conference for aeronautics and aerospace science. Spain 
4. Shi S, Xu S, Zhang B, Ma Q, Zhang Z (2019) Global second-order sliding mode control for nonlinear uncertain systems. Int $J$ Robust Nonlinear Control 29(1):224-237

5. Yang B, Yu T, Shu H, Dong J, Jiang L (2018) Robust sliding-mode control of wind energy conversion systems for optimal power extraction via nonlinear perturbation observers. Appl Energy 210:711-723

6. Wang Y, Gao Y, Karimi HR, Shen H, Fang Z (2017) Sliding mode control of fuzzy singularly perturbed systems with application to electric circuit. IEEE Trans Syst Man Cybern Syst 48(10):1667-1675

7. Moghanloo D, Ghasemi R (2016) Observer based fuzzy terminal sliding mode controller design for a class of fractional order chaotic nonlinear systems. Int J Eng Trans B Appl 29(11):1574-1581

8. Levant A, Alelishvili L (2007) Integral high-order sliding modes. IEEE Trans Autom Control 52(7):1278-1282

9. Mobayen S, Tchier F, Ragoub L (2017) Design of an adaptive tracker for $n$-link rigid robotic manipulators based on supertwisting global nonlinear sliding mode control. Int J Syst Sci 48(9):1990-2002

10. Levant A (2010) Chattering analysis. IEEE Trans Autom Control 55(6):1380-1389

11. Edwards INaC (2014) A multivariable super-twisting sliding mode approach. Automatica 50(3):984-988

12. Feng $Y, Y u X$, Man $Z$ (2002) Non-singular terminal sliding mode control of rigid manipulators. Automatica 38(12):2159-2167

13. Feng $Y, Y u X, \operatorname{Han} F$ (2013) On nonsingular terminal sliding-mode control of nonlinear systems. Automatica 49(6):1715-1722

14. Yang J-M, Kim J-H (1999) Sliding mode control for trajectory tracking of nonholonomic wheeled mobile robots. IEEE Trans Robot Autom 15(3):578-587

15. Elmokadem T, Zribi M, Youcef-Toumi K (2016) Trajectory tracking sliding mode control of underactuated AUVs. Nonlinear Dyn 84(2):1079-1091

16. Wang N, Su S-F, Pan X, Yu X, Xie G (2018) Yaw-guided trajectory tracking control of an asymmetric underactuated surface vehicle. IEEE Trans Ind Inform 15(6):3502-3513

17. Han Y, Liu X (2016) Higher-order sliding mode control for trajectory tracking of air cushion vehicle. Opt Int J Light Electron Opt 127(5):2878-2886

18. Tunstel E, Hockemeier S, Jamshidi M (1994) Fuzzy control of a hovercraft platform. Eng Appl Artif Intell 7(5):513-519

19. Wang C-I, Zhang H-y, Fu M-y (2012) Motion control of an amphibious hovercraft based on fuzzy weighting. In: 2012 IEEE 14th international conference on communication technology. IEEE, pp 1006-1011

20. Tanaka K, Iwasaki M, Wang HO (2000) Stability and smoothness conditions for switching fuzzy systems. In: Proceedings of the 2000 American Control Conference. ACC (IEEE Cat. No. 00CH36334), vol 4. IEEE, pp 2474-2478

21. Tanaka K, Iwasaki M, Wang HO (2000) Stable switching fuzzy control and its application to a hovercraft type vehicle. In: Ninth IEEE international conference on fuzzy systems. FUZZ-IEEE 2000 (Cat. No. 00CH37063), vol 2. IEEE, pp 804-809

22. Shojaei K (2015) Neural adaptive robust control of underactuated marine surface vehicles with input saturation. Appl Ocean Res 53:267-278

23. Duan K, Fong S, Zhuang Y, Song W (2018) Artificial neural networks in coordinated control of multiple hovercrafts with unmodeled terms. Applied Sciences 8(6):862

24. Fantoni I, Lozano R (2002) Non-linear control for underactuated mechanical systems. Springer, Berlin
25. Aguiar AP, Cremean L, Hespanha JP (2003). Position tracking for a nonlinear underactuated hovercraft: Controller design and experimental results. In: Proceedings of the 42 nd conference decision and control

26. Marconett AL (2003). A study and implementation of an autonomous control system for a vehicle in the zero drag environment of space. Ph.D., California, Davis

27. Wang CL, Liu ZY, Fu MY, Bian XQ (2010) Amphibious hovercraft course control based on adaptive multiple model approach. In: 2010 IEEE international conference on mechatronics and automation

28. Grau JJ (2012) Testing bench design and tilting propeller system identification for the development and control of thrustvectoring hovercraft. Ph.D., South Dakota School of Mines and Technology, Rapid City

29. Hamdy M, Shalaby R, Sallam M (2018) A hybrid partial feedback linearization and deadbeat control scheme for a nonlinear gantry crane. J Frankl Inst 355(14):6286-6299

30. Hamdy M, Shalaby R, Sallam M (2019) Experimental verification of a hybrid control scheme with chaotic whale optimization algorithm for nonlinear gantry crane: a comparative study. ISA Trans. https://doi.org/10.1016/j.isatra.2019.08.060

31. Hamdy M, Hamdan I (2014) Non-fragile controller design for a class of multivariable bilinear systems. IMA J Math Control Inf 33(2):441-455

32. Hamdy M, Hamdan I (2015) Robust fuzzy output feedback controller for affine nonlinear systems via T-S fuzzy bilinear model: CSTR benchmark. ISA Trans 57:85-92

33. Hamdy M, Hamdan I (2014) A new calculation method of feedback controller gain for bilinear paper-making process with disturbance. J Process Control 24(9):1402-1411

34. Fu M, Gao S, Wang C, Li M (2018) Human-centered automatic tracking system for underactuated hovercraft based on adaptive chattering-free full-order terminal sliding mode control. IEEE Access 6:37883-37892

35. Xie W, Cabecinhas D, Cunha R, Silvestre C (2018) Robust motion control of an underactuated hovercraft. IEEE Trans Control Syst Technol 27(5):2195-2208

36. Ding F, Meng X, Zhang T (2017) Based on disturbance observer of air cushion vehicle course sliding backstepping control. In: 2017 IEEE international conference on mechatronics and automation (ICMA)

37. Chwa D, Jeong S (2018) Coupled multiple sliding-mode control for robust trajectory tracking of hovercraft with external disturbances. IEEE Trans Ind Electron 65(5):4103-4113

38. Sira-Ramírez H (2002) Dynamic second-order sliding mode control of the hovercraft vessel. IEEE Trans Control Syst Technol 10(6):860-865

39. Defoort M, Floquet T, Kokosy A, Perruquetti W (2009) A novel higher order sliding mode control scheme. Syst Control Lett 58(2):102-108

40. Karami-Mollaee A (2016) Adaptive fuzzy dynamic sliding mode control of nonlinear systems. Int J Eng Trans B Appl 29(8):1075-1086

41. Liu X, Han Y (2014) Finite time control for MIMO nonlinear system based on higher-order sliding mode. ISA Trans 53(6):1838-1846

42. Lin $X$, Wang L (2017) Heading control of air cushion vehicle with disturbance observer based on terminal sliding mode. In 2017 IEEE International conference on mechatronics and automation (ICMA). IEEE, pp 858-862 
43. Zuo Z (2014) Non-singular fixed-time terminal sliding mode control of non-linear systems. IET Control Theory Appl 9(4):545-552

44. Cabecinhas D, Batista P, Oliveira P, Silvestre C (2017) Hovercraft control with dynamic parameters identification. IEEE Trans Control Syst Technol 26(3):785-796

45. Slotine J-JE, Li W (1991) Applied nonlinear control, vol 1. Prentice Hall, Englewood Cliffs
46. Sanders R (2003) Control of a model sized hovercraft. DCT rapporten, vol 2003

Publisher's Note Springer Nature remains neutral with regard to jurisdictional claims in published maps and institutional affiliations. 